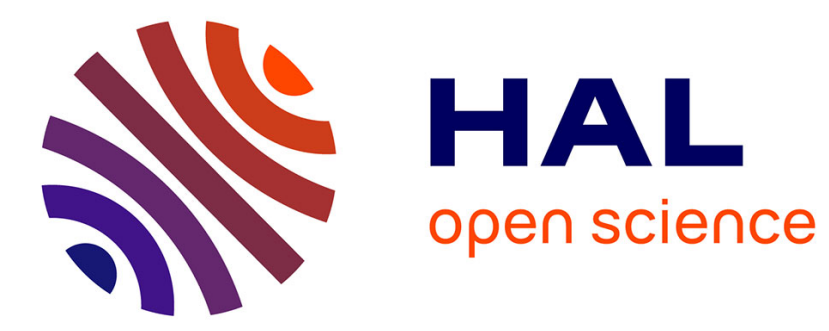

\title{
Polynomial surfaces interpolating arbitrary triangulations
}

Stefanie Hahmann, Georges-Pierre Bonneau

\section{To cite this version:}

Stefanie Hahmann, Georges-Pierre Bonneau. Polynomial surfaces interpolating arbitrary triangulations. IEEE Transactions on Visualization and Computer Graphics, 2003, 9 (1), pp.99-109. 10.1109/TVCG.2003.1175100 . hal-00319652

\section{HAL Id: hal-00319652 \\ https://hal.science/hal-00319652}

Submitted on 9 Sep 2008

HAL is a multi-disciplinary open access archive for the deposit and dissemination of scientific research documents, whether they are published or not. The documents may come from teaching and research institutions in France or abroad, or from public or private research centers.
L'archive ouverte pluridisciplinaire HAL, est destinée au dépôt et à la diffusion de documents scientifiques de niveau recherche, publiés ou non, émanant des établissements d'enseignement et de recherche français ou étrangers, des laboratoires publics ou privés. 


\section{Polynomial surfaces interpolating arbitrary triangulations}

Stefanie Hahmann*

Laboratoire LMC-IMAG

University of Grenoble

B.P. 53

F-38041 Grenoble cedex 9, France

Tel: (+33) 476635788

Fax: (+33) 476631263

email: Stefanie.Hahmann@imag.fr

\section{Georges-Pierre Bonneau}

Laboratoire iMAGIS-GRAVIR

INRIA Rhône-Alpes

655, avenue de l'Europe

F-38330 Montbonnot, France

email: Georges-Pierre.Bonneau@imag.fr
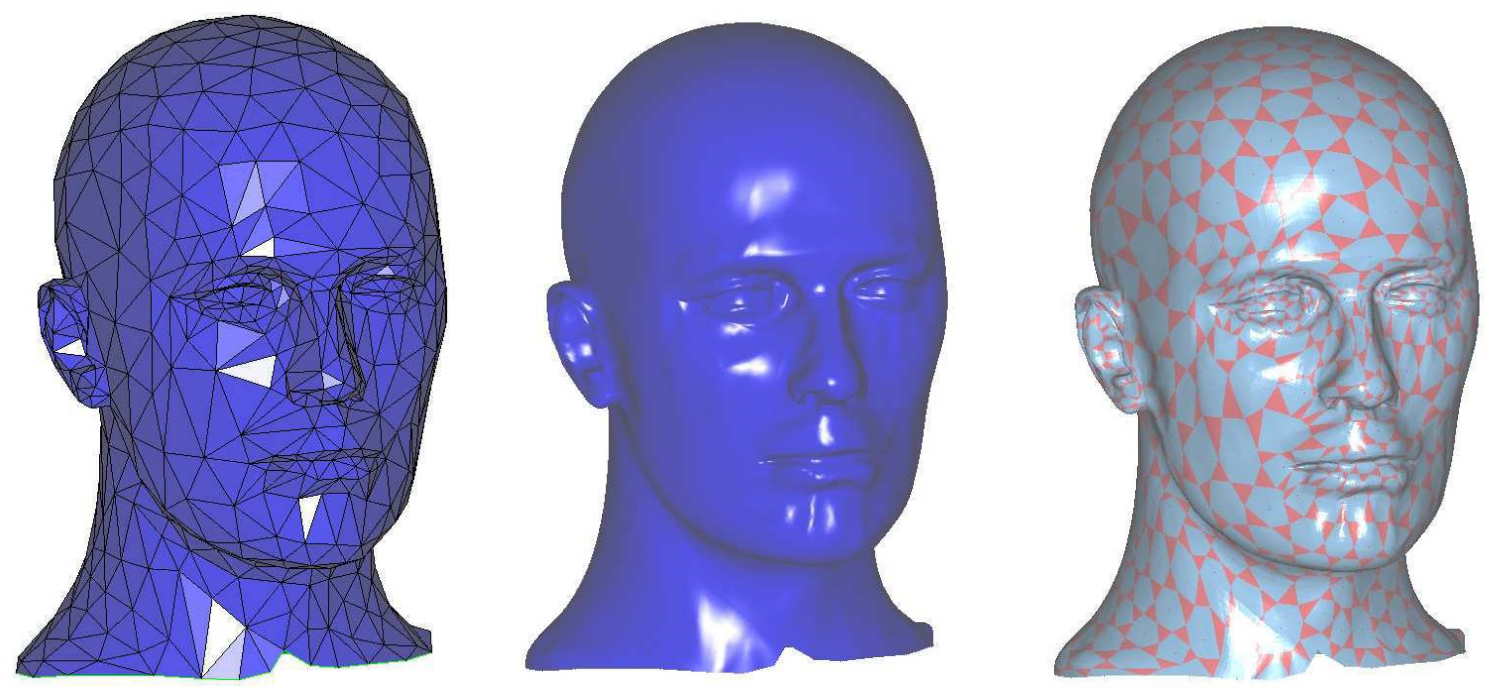

Figure 1: Mannequin, mesh left, $G^{1}$ surface middle, control-polygons right.

* corresponding author 


\begin{abstract}
Triangular Bézier patches are an important tool for defining smooth surfaces over arbitrary triangular meshes. The previously introduced 4-split method interpolates the vertices of a 2-manifold triangle mesh by a set of tangent plane continuous triangular Bézier patches of degree five. The resulting surface has an explicit closed form representation and is defined locally. In this paper, we introduce a new method for visually smooth interpolation of arbitrary triangle meshes based on a regular 4-split of the domain triangles. Ensuring tangent plane continuity of the surface is not enough for producing an overall fair shape. Interpolation of irregular control-polygons, be that in $1 D$ or in $2 D$, often yields unwanted undulations. Note that this undulation problem is not particular to parametric interpolation, but also occur with interpolatory subdivision surfaces. Our new method avoids unwanted undulations by relaxing the constraint of the first derivatives at the input mesh vertices: the tangent directions of the boundary curves at the mesh vertices are now completely free. Irregular triangulations can be handled much better in the sense that unwanted undulations due to flat triangles in the mesh are now avoided.
\end{abstract}

Keywords. triangulation, irregular 3D meshes, arbitrary topology, modeling, surfaces, triangular patches, piecewise polynomial patches, interpolation, arbitrary tangent vectors, reconstruction. 


\section{INTRODUCTION}

The easiest way of modeling free-form surfaces is to use tensor-product Bézier/BSpline or NURBS patches. Tensor-product Nurbs patches have long ago become a de-facto standard in CAD/CAM industry. But tensor-product patches are able to model only a very restricted type of surfaces, those which topological type is the same as that of a square. Unfortunately 2-manifold surfaces with arbitrary topological type are very common in everyday life. For example a cup of coffee has the topological type of a torus. Modeling the skin of a cup of coffee as a single smooth surface is not an easy task. If you are restricted to tensor-product patches, most probably you would use two pieces: one for the container and one for the handle. You would then trim the container along the joint between the two pieces. You would carefully modify the control points of the handle in order to blend as smoothly as possible the two pieces. Trimming is the common method used for modeling surfaces of arbitrary topological type with tensor-product patches. But dealing with trimmed models is rather cumbersome. These reasons explain why researchers have tried to develop mathematical models that can deal with control-polygons of arbitrary topological type, not only tensor-product control-polygons.

Two different research directions have been pursued. One is based on subdivision surfaces, that recursively subdivide the control-mesh. The other direction consists in building a patchwork of smoothly joined parametric patches, with the same topology as the control-polygon. The present paper deals with this last kind of surfaces. Not surprisingly the two directions have encountered the same main difficulty: dealing with the smoothness of the surface. Here we ensure tangent-plane continuity of the resulting surface. Our method builds a network of triangular Bézier patches that interpolates a given 2-manifold triangular mesh with arbitrary topological type. Interpolation is a very useful and intuitive feature in modeling. But unfortunately it is also quite tricky to find smooth curves or surfaces that interpolate a given control-polygon. In particular if the control-polygon has non-regular features, one small edge joined to a long edge in the $1 \mathrm{D}$ case, or one very flat triangle joined with a big triangle in the $2 \mathrm{D}$ case, then most probably the interpolating curve or surface will suffer from severe undulations in this area. Note that this problem is not particular to the parametric methods. The methods based on subdivision surfaces have exactly the same problem when it comes to interpolating control-polygons.

In this paper we introduce a new interpolation method that avoids undulations, even when interpolating irregular triangulations. Each input triangle is regularly subdivided into 4 sub-triangles, and one degree 5 Bézier patch is associated to each of the sub-triangles. These 4 Bézier patches are referred to as a macro-patch. Inside a macro-patch, the 4 Bézier patches are connected with $C^{1}$ continuity. The macro-patches are themselves connected with $G^{1}$ continuity. The key difference with our previously introduced 4-split [12] method lies in the fact that our new interpolant allows free choice of all first derivatives at each input vertex, along each input edge. Whereas the previous method was restricted to having first derivatives that form an affine transformation of a regular n-gone, this new method 
does not impose any constraint on the first derivatives. This allows to avoid any unwanted undulations when interpolating irregular triangulations.

The paper is organized as follows. After some notations and some basics about tangent plane continuity, the presentation of previous related works will then outline the main difference to our method which we believe is able to increase the general quality of such interpolating spline surfaces. Then starts the description of the algorithm (sect. 3) which is mainly composed of thee steps. The first two steps (sect. $3.2-3.4$ ) are coupled. They consist of constructing a curve network interpolating the mesh vertices and of constructing the tangent ribbons along that curves in order to satisfy the $\mathrm{G}^{1}$ conditions between adjacent patches. Step 3 (sect. 3.5) finally counts the remaining Bézier control points of the patches and explains how to calculate them. We then go into the detail of some design and computational aspects (sect. 4) of that method. Sect. 5 will present results and compare them to earlier works. The concluding remarks finally indicate some directions for further research.

\section{BASICS AND RELATED WORKS}

\subsection{Notations}

The problem we address is to find a parametric polynomial surface which interpolates a given triangulated surface mesh $\mathcal{M}$ with tangent plane continuity. The triangle mesh can be of arbitrary topology but should be 2-manifold. There is no restriction on the valence of its vertices. It can be an open or a closed mesh. The requirements on the surface are to

- be polynomial of as low degree as possible,

- interpolate the mesh vertices,

- be defined locally and

- be smooth in the sense of having a pleasing shape.

The smoothness requirement is a global one, and it conflicts therefore with a local definition of the surface. We therefore try to get an overall well shaped surface only by doing local operations.

The general procedure consists of constructing parametric patches in one-to-one correspondence to the mesh faces. Each patch boundary curve corresponds to an edge of $\mathcal{M}$ and interpolates both end points. Each patch is in our case a polynomial image of the unit domain triangle. The polynomial patches and curves are represented in BernsteinBézier basis. A curve of degree $n$ with control-points $\boldsymbol{b}_{0}, \cdots, \boldsymbol{b}_{n}$ has the parametric equation $B(t)=\sum_{i=0}^{n} \boldsymbol{b}_{i} t^{i}(1-t)^{n-i}$. A triangular patch of degree $n$ with control-points $\boldsymbol{b}_{(i, j, k), i+j+k=n}$ has the parametric equation $S(s, t)=\sum_{(i, j, k), i+j+k=n} \boldsymbol{b}_{(i, j, k)} s^{i} t^{j}(1-s-t)^{k}$. 

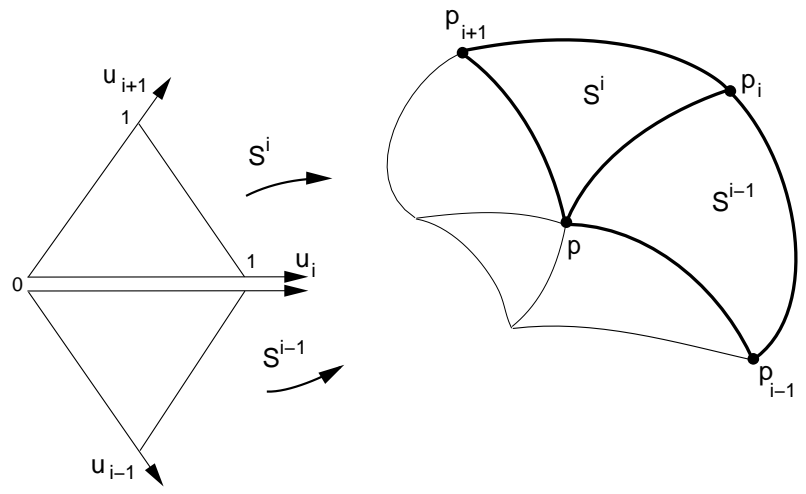

Figure 2: parameterization.

The patches of the surface we aim to construct are defined locally around the vertices which they interpolate. We adopt therefore the following parameterization. Let us consider a mesh vertex $\boldsymbol{p} \in \mathbb{R}^{3}$ and its neighborhood points $\boldsymbol{p}_{1}, \ldots, \boldsymbol{p}_{n}$ ordered in a trigonometric sense. The integer $n$ is called valence of $\boldsymbol{p}$. The patches around the vertex are numbered $S^{1}$ to $S^{n}$. Let $u_{i} \in[0,1]$ be the parameter corresponding to the curve between $\boldsymbol{p}$ and $\boldsymbol{p}_{i}$, the patch $S^{i}$ is then parametrized as shown in fig. 2 . In the following we denote $\Gamma_{i}\left(u_{i}\right)$ the patch boundary curve joining $\boldsymbol{p}$ to $\boldsymbol{p}_{i}$ :

$$
\Gamma_{i}\left(u_{i}\right):=S^{i}\left(u_{i}, 0\right)=S^{i-1}\left(0, u_{i}\right) \quad u_{i} \in[0,1] .
$$

\subsection{Tangent plane continuity}

We require our interpolating surface to be tangent plane - e.g. $\mathrm{G}^{1}$ - continuous. Tangent plane continuity doesn't depend on parameterization as $\mathrm{C}^{1}$ continuity does. Tangent plane continuity is the mostly used definition of first order continuity for free-form parametric surfaces in CAGD. In addition to being parameter-independent, it also allows more free parameters in comparison to the $\mathrm{C}^{1}$ continuity.

The surface should be $\mathrm{G}^{1}$ continuous which means to have continuously varying tangent planes between the patches. The $\mathrm{G}^{1}$ conditions are at the origin of every step in the surface construction algorithm. They have to be satisfied between adjacent patches and at the mesh vertices where an arbitrary number of patches meet. They therefore have to be satisfied by the boundary curves at the mesh vertices and by the cross-boundary tangents.

Let $S^{i}$ and $S^{i-1}$ be two adjacent patches sharing a common boundary curve $\Gamma_{i}\left(u_{i}\right)$. They meet with tangent plane continuity if there exist three scalar functions $\Phi_{i}, \mu_{i}, \nu_{i}$ such that

$$
\Phi_{i}\left(u_{i}\right) \frac{\partial S^{i}}{\partial u_{i}}\left(u_{i}, 0\right)=\mu_{i}\left(u_{i}\right) \frac{\partial S^{i}}{\partial u_{i+1}}\left(u_{i}, 0\right)+\nu_{i}\left(u_{i}\right) \frac{\partial S^{i-1}}{\partial u_{i-1}}\left(0, u_{i}\right)
$$

where $\mu_{i}\left(u_{i}\right) \nu_{i}\left(u_{i}\right)>0$ (preservation of orientation) and $\frac{\partial S^{i}}{\partial u_{i+1}}\left(u_{i}, 0\right) \times \frac{\partial S^{i-1}}{\partial u_{i-1}}\left(0, u_{i}\right) \neq 0$ (well defined normal vectors). This formula means that the three partial derivatives along the boundary curve $\Gamma_{i}$ are always coplanar, see fig. 3 . 


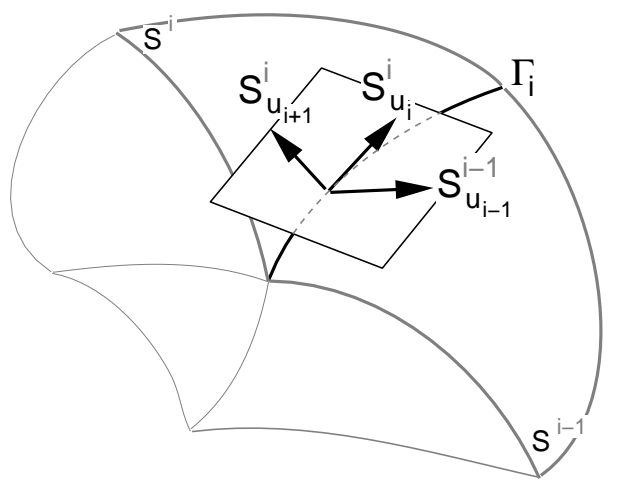

Figure 3: Tangent plane continuity.

The goal is now to determine for each mesh vertex of valence $n$ the scalar valued functions $\Phi_{i}, \mu_{i}, \nu_{i}$ and the patches $S^{i}, i=1, \ldots, n$. Of course there is an infinite number of solutions. We require the patches to be polynomial with all advantages they have with respect to rational patches. Also we require them to be of minimal possible degree and to be defined explicitly and locally, meaning that we don't want to have to solve any global linear system in order to compute them.

\subsection{Related works}

The earliest interpolation schemes are due to Piper [22], Shirman, Séquin [24] and some others $[14,17]$ which are all based on the same idea using a Clough-Tocher domain split. The initial work to that can be found in [6]. They all apply to arbitrary triangle meshes and result in three polynomial patches of degree four per mesh face. The problem of polynomial $\mathrm{G}^{1}$ interpolation is, that the surfaces have to be twist compatible at the vertices. The problem is solved by splitting the domain triangle Clough-Tocher like into three triangles by introducing a new point at the interior. Due to the very low degree only few degrees of freedom are available for shape control. This can be the reason for that what Mann et al. found out in their survey paper [17]. They compared polynomial and rational triangular interpolation schemes and conclude, that all of them suffer from shape defects.

Then Loop [15] developed another polynomial scheme which results in only one patch for a mesh face. Its low degree six is obtained by choosing a special setting for the scalar functions $\Phi_{i}, \mu_{i}, \nu_{i}$ in equation (1), namely

Loop'94:

$\Phi_{i}$ function of degree 2

$$
\mu_{i}=\nu_{i} \equiv \frac{1}{2}
$$

in order to solve the twist problem. This particular choice fixes entirely the patch boundary curves up to a scalar degree of freedom per vertex. He obtains however quite fair surfaces by relaxing the interpolation condition. It belongs therefore not really to the class of schemes we consider here, but the way he solved the twist problem, motivated our work.

A third type of methods is the basic 4-split method [12]. 


$$
\begin{array}{ll}
\text { Hahmann/Bonneau'00: } & \Phi_{i} \text { piecewise function of degree } 1 \\
& \mu_{i}=\nu_{i} \equiv \frac{1}{2} .
\end{array}
$$

By taking Loop's low degree setting for the scalar functions $\mu_{i}, \nu_{i}$ and by lowering the degree of $\Phi_{i}$ by one and by introducing piecewise polynomial boundary curves and crossboundary tangents, we obtained enough degrees of freedom for the curve network construction. The four patches (due to the domain triangle 4-split) are of degree five and interpolate the mesh vertices.

It turns out that this particular setting of $\mu_{i}=\nu_{i}=$ const can lead to "misbehavior" of the surface, to unpleasant shapes if the input mesh contains irregularities. Irregularities mean for example that very short and very long edges meet at the same vertex, or that small flat triangles are joint to more equiangular triangles. All these situations in an input mesh make it extremely difficult to find an interpolating smooth surface. The same problem occurs also for interpolatory subdivision surfaces, they also are not able to result in a fair interpolating surface in that case.

One solution to that problem can consist in a kind of preprocessing step of the input mesh by trying to optimize the mesh with respect to one well chosen cost function. However, in this case interpolation doesn't make sense anymore.

What we want to develop in the present paper is a new mesh interpolation method which allows for arbitrary values of the scalar functions around a vertex, while maintaining at the same time the interpolation scheme polynomial of degree five. The functions will be linear. What really is the difference between using constant or arbitrary linear functions will be explained in detail in the following section.

Other triangular interpolants exist including the convex combination schemes $[8,9$, 11, 19], boundary curve schemes [21], algebraic methods [2], singular parameterization [18], quasi $\mathrm{G}^{1}$ surfaces [16], and methods for meshes with restricted vertex valences [25].

\section{THE $\mathrm{G}^{1}$ INTERPOLATION SCHEME}

The algorithm of the present method consists mainly of three constructive steps:

(1) boundary curve network,

(2) cross-boundary tangents,

(3) fill-in patches.

The first two steps are linked together because of the $\mathrm{G}^{1}$ conditions. First we examine in detail the $\mathrm{G}^{1}$ conditions at a mesh vertex. We get first and second derivatives of the patch boundary curves at the vertices. These data are then used to find a curve network interpolating these data and which is therefore $\mathrm{G}^{1}$ compatible. 


\subsection{Tangent plane continuity at mesh vertices}

The tangent plane continuity conditions at a mesh vertex of valence $n$ consist of the $n$ equations (1) between the $n$ patches meeting at that vertex evaluated at the parameters $u_{i}=0$ :

$$
\Phi_{i}(0) \Gamma_{i}^{\prime}(0)=\mu_{i}(0) \Gamma_{i+1}^{\prime}(0)+\nu_{i}(0) \Gamma_{i-1}^{\prime}(0), \quad i=1, \ldots, n .
$$

The indices are taken modulo $n$. It turns out that at the vertex, the three partial derivatives in (1) are identical to the first derivatives of the three boundary curves $\Gamma_{i-1}, \Gamma_{i}$, and $\Gamma_{i+1}$, see fig. 4 .

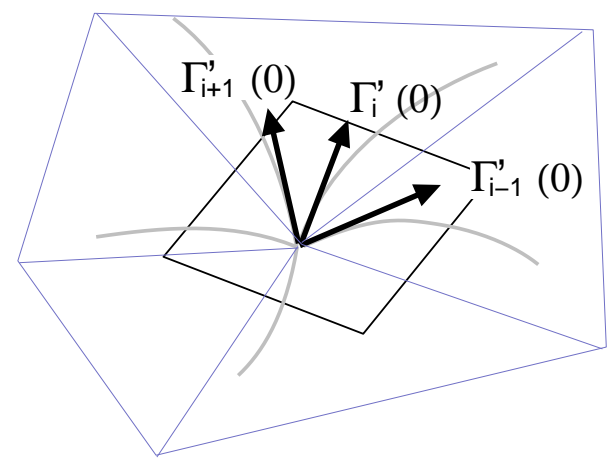

Figure 4: Tangent plane continuity at mesh vertex.

Equation (2) relates therefore the values of the scalar functions around a vertex to the first derivatives of the boundary curves. From the general $\mathrm{G}^{1}$ condition we already know that the degree of the patches is related to the degree of the scalar functions. If one wants to get patches of as low degree as possible, one has to keep the degree of these functions as low as possible. This was done in $[15,12]$, where $\mu_{i}=\nu_{i}=$ const. It was shown in these papers that in the case of $\mu_{i}=\nu_{i}=$ const the first derivatives in terms of Bézier control points always form an affine transformation of a regular planar n-gon. This leads to very restricted positions of the derivative vectors around a vertex. There is not enough freedom for choosing the derivatives of the curves at the vertex. Indeed, in order to get smooth surfaces it is very important first to build smooth boundary curves, as it was pointed out by S. Mann in [17], and hence to be able to choose the first derivatives with as most freedom as possible. Fig. 5 (left) shows a top-view zoom on the real example given later in fig. 15, 16 and 17 (right), centered on the order six vertex. The tangents corresponding to $\mu_{i}=\nu_{i} \equiv$ const are shown in fig. 5 (right). Because of the restriction on their choice, these tangents lead to boundary curves with undulations, and thus to unpleasant shapes as can been seen in fig. 16 right. On the contrary, our new method enables to choose arbitrary tangents, as shown in fig. 5 (middle), and thus to remove boundary curve undulations, as shown in fig. 17 (right).

\section{Conclusion:}

The setting of $\mu_{i}=\nu_{i} \equiv$ const doesn't allow for an arbitrary choice of the boundary curve's derivatives at the mesh vertices. However, it works well if the input mesh is almost 
regular in the sense of almost equiangular triangles. A preprocessing mesh optimization would make sense if interpolation of the control mesh is not required.

\subsection{Collecting data ensuring $\mathrm{G}^{1}$ continuity at mesh vertices}

\section{Tangents}

For simplification, let us first introduce the following notation for the tangents (first derivatives) of the boundary curves at a mesh vertex:

$$
\boldsymbol{d}_{i}^{1}:=\Gamma_{i}^{\prime}(0)=\frac{\partial S^{i}}{\partial u_{i}}(0,0)
$$

Let us now explain how to determine the quantities which are related by equation (2), namely $\mu_{i}(0), \nu_{i}(0), \Phi_{i}(0)$ and the curve's tangents $\boldsymbol{d}_{i}^{1}, i=1, \ldots, n$.

If one multiplies (cross product) for each index $i$ the $G^{1}$ equation $\Phi_{i}(0) \boldsymbol{d}_{i}^{1}-\mu_{i}(0) \boldsymbol{d}_{i+1}^{1}-$ $\nu_{i}(0) \boldsymbol{d}_{i-1}^{1}=0$ by the vectors $\boldsymbol{d}_{i-1}^{1}, \boldsymbol{d}_{i}^{1}, \boldsymbol{d}_{i+1}^{1}$ resp., one gets three vector valued equations which are then multiplied (dot product) each by the vector $\boldsymbol{n}$ (normal to the $\boldsymbol{d}_{i}^{1}$ ) one gets a $(3 \times 3)$ linear system of equations of rank 2 . Doing so for each index $i$, one gets the following formulas

$$
\mu_{i}(0)=\frac{\left|\boldsymbol{d}_{i}, \boldsymbol{d}_{i+1}, \boldsymbol{n}\right|}{\left|\boldsymbol{d}_{i-1}, \boldsymbol{d}_{i+1}, \boldsymbol{n}\right|} \Phi_{i}(0), \quad \nu_{i}(0)=\frac{\left|\boldsymbol{d}_{i-1}, \boldsymbol{d}_{i}, \boldsymbol{n}\right|}{\left|\boldsymbol{d}_{i-1}, \boldsymbol{d}_{i+1}, \boldsymbol{n}\right|} \Phi_{i}(0), \quad i=1, \ldots, n
$$

The tangents of the boundary curves can therefore be chosen arbitrarily in length and direction as long as they belong to the same plane, namely the tangent plane. Then the scalar values $\mu_{i}(0)$ and $\nu_{i}(0)$ are also fixed up to a scalar factor $\Phi_{i}(0)$ by equations $(3)$. Similar formulas have been developed by W. Du in [4], but his $\mathrm{G}^{1}$ surface is then composed of rational patches.

In practice we choose the normalization factor $\Phi_{i}(0)$ such that $\mu_{i}(0) \cdot \nu_{i}(0)=\frac{1}{4}$. This choice is motivated by the need of a normalization that generalizes the regular case where $\mu_{i}(0)=\nu_{i}(0)=1 / 2$. And it will simplify the computation of the boundary curves where this product occurs??.

Fig. 5 (left) shows a zoom on a real example of a vertex with six common edges. An arbitrary choice of the curves' tangents leads to the Bézier control points of the boundary curves as shown in fig. 5 (middle). They are obtained by the new method which is described in section 4 . The tangent control points corresponding to constant scalar functions, $\mu_{i}(0)=$ $\nu_{i}(0) \equiv \frac{1}{2}$, are shown in fig. 5 (right). The edges in the background show the deviation in that case. 

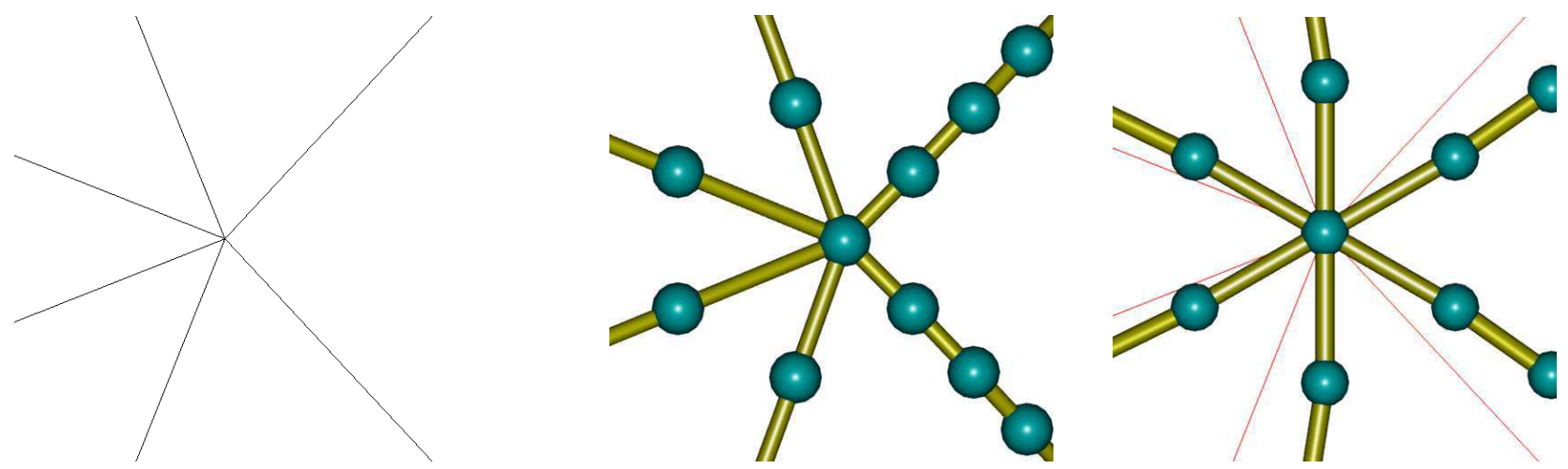

Figure 5: Left: zoom on a $3 D$ vertex configuration with six common edges. Middle: Arbitrary tangents at mesh vertex. The vertex together with the curves' Bézier control points build the polygons. Right: tangent Bézier control points corresponding to a constant value of the scalar functions, in particular $\mu_{i}(0)=\nu_{i}(0) \equiv \frac{1}{2}$.

\section{Geometrical interpretation:}

Formulas (3) have the following geometrical meaning: for each triple of tangents $\left\{\boldsymbol{d}_{i-1}^{1}, \boldsymbol{d}_{i}^{1}\right.$, $\left.\boldsymbol{d}_{i+1}^{1}\right\}$ the value of $\mu_{i}(0)$ is proportional to the area of the triangle $\Delta\left(\boldsymbol{p}, \boldsymbol{p}+\boldsymbol{d}_{i}^{1}, \boldsymbol{p}+\boldsymbol{d}_{i+1}^{1}\right)$ and $\nu_{i}(0)$ is proportional to the area of the triangle $\Delta\left(\boldsymbol{p}, \boldsymbol{p}+\boldsymbol{d}_{i-1}^{1}, \boldsymbol{p}+\boldsymbol{d}_{i}^{1}\right)$, see fig. 6 .

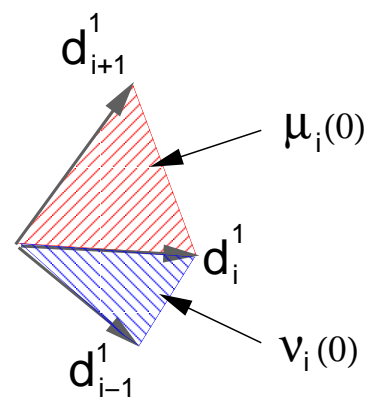

Figure 6: Geometrical meaning of scalar functions. The values of $\mu_{i}(0), \nu_{i}(0)$ are proportional to the area of the shaded triangles.

\section{Scalar functions:}

Once the values (3) are determined for each mesh vertex, the scalar functions can be taken (piecewise) linear along the domain triangle edges where they are defined on:

$$
\text { (piecewise) linear functions: } \quad \mu_{i}, \nu_{i}, \Phi_{i}:[0,1] \rightarrow \mathbb{R} \text {. }
$$

\section{Twists}

The second derivatives of the patch boundary curves are also involved when establishing $\mathrm{G}^{1}$ continuity at a mesh vertex. This can be seen when differentiating (1) with respect to $u_{i}$ and evaluating it at $u_{i}=0, i=1, \ldots, n$. One obtains the following necessary system of conditions of $\mathrm{G}^{1}$ continuity at the mesh vertices:

$$
\Phi_{i}(0) \boldsymbol{d}_{i}^{2}=\mu_{i}^{\prime}(0) \boldsymbol{d}_{i-1}^{1}-\Phi_{i}^{\prime}(0) \boldsymbol{d}_{i}^{1}+\nu_{i}^{\prime}(0) \boldsymbol{d}_{i+1}^{1}+\mu_{i}(0) \boldsymbol{t}_{i-1}+\nu_{i}(0) \boldsymbol{t}_{i}, \quad i=1, \ldots, n,
$$


where $\boldsymbol{d}_{i}^{2}:=\Gamma_{i}^{\prime \prime}(0)=\frac{\partial^{2} S^{i}}{\partial u_{i}^{2}}(0,0)$ denote the second boundary curves derivatives at the vertex, and $\boldsymbol{t}_{i}:=\frac{\partial^{2} S^{i}}{\partial u_{i} \partial u_{i+1}}(0,0)$ denote the twist vector of patch $S^{i}$ at the vertex. The twists and the second derivatives are the unknowns in these equations. The best way to proceed is to choose all the twists $\boldsymbol{t}_{1}, \ldots, \boldsymbol{t}_{n}$ and then simply evaluate the equations separately from each other in order to get values for the second derivative $\boldsymbol{d}_{i}^{2}$. No linear system has to be solved for that. How the twists are chosen will be explained in section 4 .

\section{Conclusion:}

At each mesh vertex we now have fixed the data which is necessary to satisfy $\mathrm{G}^{1}$ continuity there:

- the position (interpolation of mesh vertices),

- the tangents,

- the second derivatives of the patch boundary curves,

- the twists of the patches surrounding that vertex.

One is therefore tempted to believe that a curve network composed of quintic Hermite curves between two neighboring vertices which interpolates these data could be chosen. Unfortunately this would generally lead to rational patches, as we will explain in the next section.

\subsection{Curves and tangent ribbons are linked}

What we call tangent ribbons are the cross-boundary derivatives along the macro-patch boundary curves. They ensure the $\mathrm{G}^{1}$ continuity between adjacent patches. An equivalent condition to (1) of $\mathrm{G}^{1}$ continuity is used for their construction.

\section{tangent ribbons:}

The patches $S^{i}$ and $S^{i-1}$ meet with $\mathrm{G}^{1}$ continuity along the common boundary, if there exist three scalar functions $\Phi_{i}, \mu_{i}, \nu_{i}$ and a vector valued function in $\mathbb{R}^{3} \boldsymbol{V}_{i}$ such that the following two equations hold:

$$
\begin{aligned}
& 2 \mu_{i}\left(u_{i}\right) \frac{\partial S^{i}}{\partial u_{i+1}}\left(u_{i}, 0\right)=\Phi_{i}\left(u_{i}\right) \Gamma_{i}^{\prime}\left(u_{i}\right)+\boldsymbol{V}_{i}\left(u_{i}\right) \\
& 2 \nu_{i}\left(u_{i}\right) \frac{\partial S^{i-1}}{\partial u_{i-1}}\left(0, u_{i}\right)=\Phi_{i}\left(u_{i}\right) \Gamma_{i}^{\prime}\left(u_{i}\right)-\boldsymbol{V}_{i}\left(u_{i}\right) .
\end{aligned}
$$

The equivalence between (5), (6) and (1) can be seen by adding up equations (5), (6). From section 3.2 it is known that $\mu_{i}$ and $\nu_{i}$ are linear functions in order to guarantee an arbitrary choice of the tangents at the vertices. This implies however that the tangent ribbons $\frac{\partial S^{i}}{\partial u_{i+1}}\left(u_{i}, 0\right)$ and $\frac{\partial S^{i-1}}{\partial u_{i-1}}\left(0, u_{i}\right)$ defined by eq. $(5),(6)$ between $S^{i}$ and $S^{i-1}$ would be in general rational. Therefore the patches would all be rational in contradiction with our main requirement: the interpolation surface must be polynomial. 


\section{boundary curves:}

In order to keep in the class of polynomial patches the boundary curves $\Gamma_{i}$ common to a mesh vertex are chosen such that they satisfy the following condition: their derivatives $\Gamma_{i}^{\prime}\left(u_{i}\right)=\frac{\partial S^{i}}{\partial u_{i}}\left(u_{i}, 0\right)$ are defined as a product of the linear scalar functions $\mu_{i}, \nu_{i}$ and a vector valued piecewise polynomial function $\boldsymbol{H}_{i}$, i.e.

$$
\Gamma_{i}^{\prime}\left(u_{i}\right):=\mu_{i}\left(u_{i}\right) \cdot \nu_{i}\left(u_{i}\right) \cdot \boldsymbol{H}_{i}\left(u_{i}\right), \quad i=1, \ldots, n
$$

The choice (7) is the only possibility that yields a polynomial solution of (5) and (6). Now, an explicit formula of the boundary curves can be given by assembling the results about the tangents and second derivatives at each vertex from sect. 3.2 subject to the polynomial condition (7) between two neighboring vertices. Each boundary curve is finally a uniquely defined piecewise $\mathbf{C}^{1}$ quintic Bézier curve $\Gamma_{i}\left(u_{i}\right), u_{i} \in[0,1]$, composed of two pieces. The two sets of control points $\left\{\boldsymbol{b}_{k}^{L}\right\}_{k=0}^{5}$ and $\left\{\boldsymbol{b}_{5-k}^{R}\right\}_{k=5}^{0}$ are shown in fig. 7 .

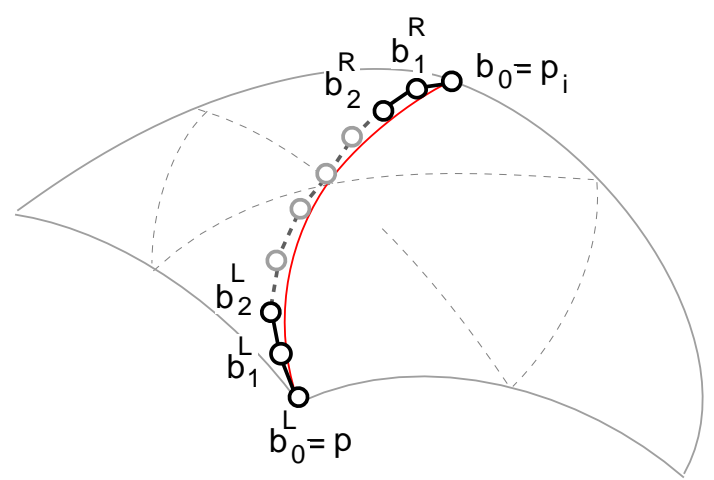

Figure 7: Bézier control points of boundary curves.

The control points $\boldsymbol{b}_{0}^{L}, \boldsymbol{b}_{1}^{L}, \boldsymbol{b}_{2}^{L}$ and $\boldsymbol{b}_{0}^{R}, \boldsymbol{b}_{1}^{R}, \boldsymbol{b}_{2}^{R}$ are determined by interpolating the position, tangent and second derivative known at the end points $\boldsymbol{p}_{k}, \boldsymbol{p}_{i}$ (mesh vertices).

The function $\boldsymbol{H}_{i}$ defines the curve's derivative by (7). It can be chosen piecewise quadratic. Its Bézier control points are denoted by $\boldsymbol{h}_{k}^{L}$ and $\boldsymbol{h}_{k}^{R}, k=0,1,2 . \boldsymbol{h}_{0}^{L}, \boldsymbol{h}_{1}^{L}, \boldsymbol{h}_{0}^{R}, \boldsymbol{h}_{1}^{R}$ are known from $\boldsymbol{b}_{0}^{L}, \boldsymbol{b}_{1}^{L}, \boldsymbol{b}_{2}^{L}$ and $\boldsymbol{b}_{0}^{R}, \boldsymbol{b}_{1}^{R}, \boldsymbol{b}_{2}^{R}$, since position and tangent of $\Gamma_{i}^{\prime}$ are already known. A comparison of the coefficients in (7) together with the two linear conditions expressing $\mathrm{C}^{1}$ continuity of $\Gamma_{i}$ at $u_{i}=1 / 2$ determine exactly the remaining unknowns.

\subsection{Compatible tangent ribbons}

The tangent ribbons which guarantee tangent plane continuity between adjacent patches have to satisfy conditions (5) and (6). The requirements on the unknown function $\boldsymbol{V} i$ are twice. $\boldsymbol{V}_{i}$ has to be compatible with the polynomial requirement. Therefore the same multiplying factor as in the case of the curve derivative (see (7)) is necessary:

$$
\boldsymbol{V}_{i}\left(u_{i}\right):=\mu_{i}\left(u_{i}\right) \cdot \nu_{i}\left(u_{i}\right) \cdot \boldsymbol{W}_{i}\left(u_{i}\right), \quad u_{i} \in[0,1]
$$


Second, at the end points (mesh vertices) $\boldsymbol{V}_{i}$ has to be compatible with the boundary curve tangents computed earlier (sect. 3.2), since the following relation holds:

$$
\frac{\partial S^{i}}{\partial u_{i+1}}(0,0)=\boldsymbol{d}_{i+1}^{1}, \quad i=1, \ldots, n
$$

The same relations hold at the opposite vertex, they fix the values of $\boldsymbol{V}_{i}$ at the end points, i.e. $\boldsymbol{W}_{i}(0), \boldsymbol{W}_{i}(1)$. Analogously, the $\mathrm{G}^{1}$ compatible twists computed earlier (sect. 3.2) at each vertex are related to the derivative of $\boldsymbol{V}_{i}$ at the end points, i.e. they fix the values of $\boldsymbol{W}_{i}^{\prime}(0), \boldsymbol{W}_{i}^{\prime}(1)$.

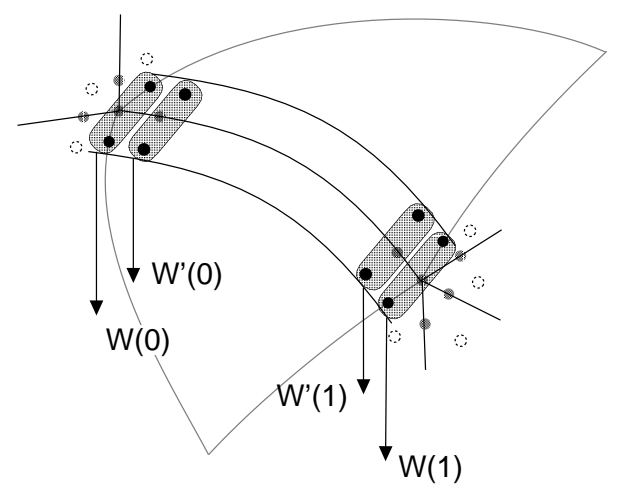

Figure 8: $G^{1}$ compatible tangent ribbons at end points.

All data we need for $\mathrm{G}^{1}$ compatible tangent ribbons are fixed now. $\boldsymbol{W}_{i}$ can interpolate these data if we choose it as a piecewise $\mathrm{C}^{0}$ quadratic or a cubic Hermite curve. $\boldsymbol{V}_{i}$ will therefore be either piecewise $\mathrm{C}^{0}$ quartic or quintic polynomial. Finally, from equ. (5), (6) together with (8) follows that the tangent ribbons will be either a piecewise $\mathrm{C}^{0}$ cubic or a quartic polynomial. Both possibilities don't increase the degree of the patches which is equal to five because of the boundary curves. An explicit Bézier representation can be computed directly from the preceding considerations.

\subsection{Constructing Bézier patches}

The third and last step of the algorithm consists of the construction of the surface patches by determining the remaining control points. How to choose them and what kind of conditions must they fulfill are the two questions to be answered now. Recall that we call the group of four Bézier patches associated to an input mesh triangle as a macro-patch (see introduction).

It turns out from sect. 3.3 that the macro patch boundary curves are piecewise quintic curves consisting of two pieces joining with $\mathrm{C}^{1}$ continuity at the parameter value $\frac{1}{2}$. The underlying parameterization for each macro patch is based on a 4-split of the domain triangle, as shown if fig. 9 . 

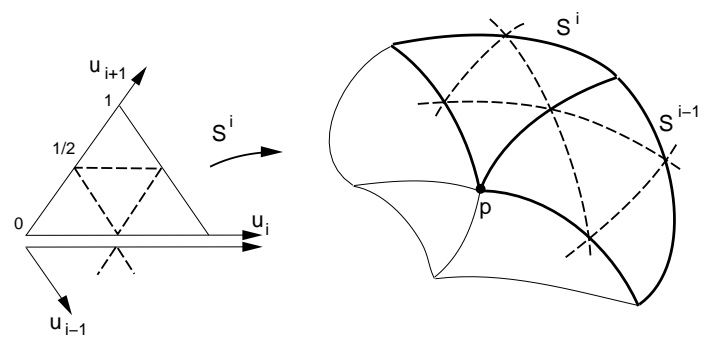

Figure 9: 4-split parameterization of the macro patches.

The macro patches are finally composed of four quintic triangular Bézier patches. From sect. 3.3 and 3.4 we know the coordinates of all the boundary and the first inner row of control points, see black dots in fig. 10 left. These data guarantee a $\mathrm{G}^{1}$ joint between all macro patches. However the remaining 15 inner control points of each macro patch are not free, because the joints between these four inner patches also need to be tangent plane continuous. In [7] it is shown that two triangular Bézier patches of the same degree join with $\mathrm{C}^{1}$ continuity along their common boundary if all pairs of sub triangles (subsets of four control points) across the common boundary are coplanar and are an affine map of the domain triangles. In the present method the patches are defined on the unit triangle which implies that each pair of sub triangles of control points must be a parallelogram, see fig. 10 right. Since this linear parallelogram equation already holds at the boundary edge midpoints, the remaining 15 inner control points are finally related across the inner boundaries by 9 linear parallelogram equations. Therefore 6 inner control points remain free for shape control.
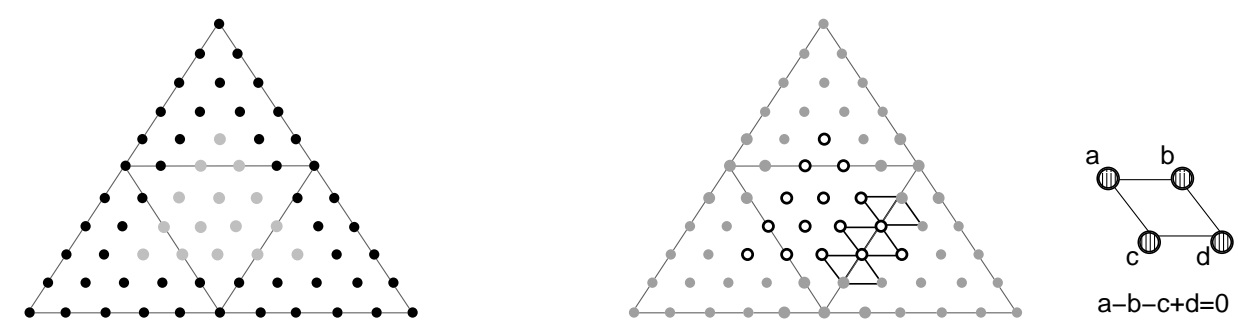

Figure 10: left: schematic representation of macro patch control points that ensure the $G^{1}$ joint between all macro patches. right: $C^{1}$ conditions for inner control points.

\section{DESIGN ISSUES}

Interpolation of arbitrary triangle meshes with the present method based on an arbitrary choice of the curve network offers a lot of degrees of freedom. They are welcome for shape control as well as for modeling complex shapes. Let us enumerate these degrees of freedom. For each mesh vertex of order $n$, there is one control point, there are $n$ tangents, and $n$ twists which are free. Then for each macro patch corresponding to a mesh face, there are six control points free. These degrees of freedom have two main advantages: First, almost all of them offer an intuitive geometrical way to fix them. Second, they are numerous enough to allow construction of globally smooth surfaces. 


\section{Some rules for fixing the degrees of freedom}

It is obvious that one input mesh can have quite different interpolating surfaces. Ideally, the surface can be seen as an enveloping skin of the mesh vertices which is more or less tight. This is equal to say that one wants a globally smooth surface. From the survey of Mann et al. [17] it is known that the globally smoothness is the most difficult challenge for all mesh interpolation schemes. Other particular design features like flat points, sharp points or corners, can of course be obtained by acting locally on the degrees of freedom. In figs. 11, 12 from left to right, three surfaces are shown which all interpolate the same input mesh (fig. 11 right).
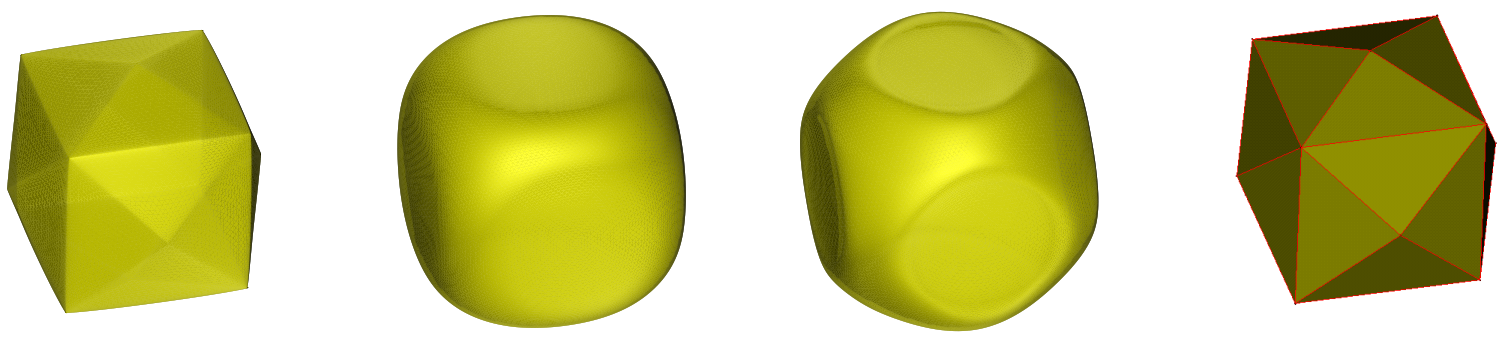

Figure 11: three interpolating surfaces — input 'cube' mesh.
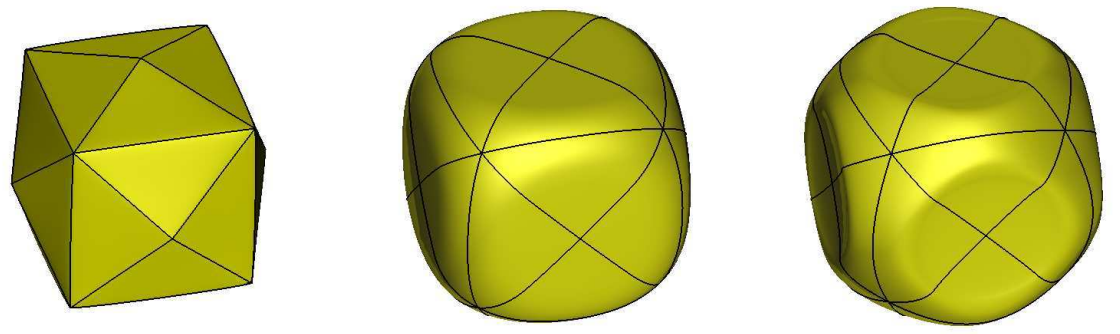

Figure 12: three interpolating surfaces with boundary curves.

All the degrees of freedom listed above can be set up by using some heuristic but geometrically based rules, such that the resulting surface generally suffices the user.

- For each mesh vertex, there is a theoretical free point, corresponding to the patch corners and the boundary curve's end points. It is set up equal to the mesh vertex in order to make the surface interpolating the mesh. However, it is always possible to fix them otherwise.

- At each vertex of valence $n$, there are $n$ tangents free to choose. Together with the second derivatives, they entirely determine the boundary curves coming in to that vertex. The curve network which is interpolated by the patches is the most important step toward a globally smooth and well pleasing surface. Since the curves are constructed in correspondence to the mesh edges, we choose the following geometrically intuitive rule to fix the tangent: as tangent vector for the edge between the vertex $\boldsymbol{p}$ and $\boldsymbol{p}_{i}$ take the unit vector in the intersection of the tangent plane at $\boldsymbol{p}$ and the plane spanned by the edge and the normal vector at $\boldsymbol{p}_{i}$ and which is scaled by an appropriate factor. The default scaling factor for each tangent is taken as proposed in [22], i.e. $\frac{4}{9}\left\|\boldsymbol{p}-\boldsymbol{p}_{i}\right\|$ for a tangent of a cubic 
curve. This scaling factor can furthermore be used as an intuitive design handle, which can easily be modified in a 3D graphics program. It is geometrically very intuitive because it governs the flatness of the surface at the vertices. The surfaces in figs. 11, 12 from left to right are obtained by simply increasing this scalar factor simultaneously for all vertices.

- At each vertex, there are $n$ twists free. They determine entirely the $n$ second derivatives of the boundary curves around the vertices, see equ. (4). The question of how to choose twists of polynomial patches has driven to a lot of publications $[1,23,20,10,3]$. Zero twists are always an easy solution, but in [7] it is shown that they never lead to satisfactory results. This can be confirmed in the present case. The lack of an intuitive heuristic rule for these values leads us to use a linear least squares minimization in order to choose them. More specifically, we minimize the following energy integral, a linearized version of a thin plate's bending energy: $\int S_{u u}+2 S_{u v}+S_{v v} d u d v$.

All the degrees of freedom which are fixed up to now, allow for the construction of uniquely defined patch boundary curves and $\mathrm{G}^{1}$ compatible cross boundary tangents (sect. $3.2-3.4)$.

- At the interior of each macro patch there are $\mathbf{6}$ inner control points free for shape control (sect. 3.5). This doesn't differ from the previously developed method [12]. We generally use the same least square energy minimization as for the computation of the twists. This is explained in more detail in [13].

\section{RESULTS}

We begin this section with examples of familiar shapes. These examples allow some comparison with earlier related works based on Clough-Tocher splitting, because these works were tested in S. Mann's state-of-the-art paper [17] using similar familiar shapes. The methods using a Clough-Tocher split [22, 24, 14] have the advantage to yield degree four polynomial patches and only three patches per macro-patch. Together with [21] they also allow for arbitrary tangents. However, the sphere and torus examples of fig. 13 and 14 compared with the results shown in Mann's paper clearly show that our method yields better results. We believe that this is mainly due to the parameterization of the surface which is smoother because of the 4-split of the triangles. This improved behaviour of the iso-parametric lines is illustrated in fig. 13 in comparison with fig. 8.8 in [17]. 

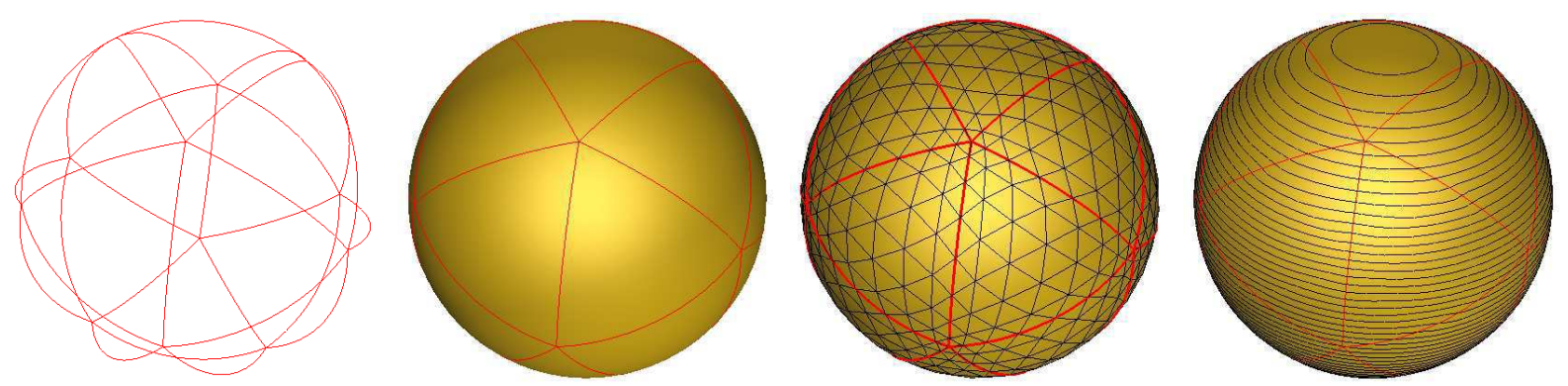

Figure 13: Icosahedron example. from left to right: boundary curves, surface with boundary curves, surface with iso-parametric lines, surface with isophotes.
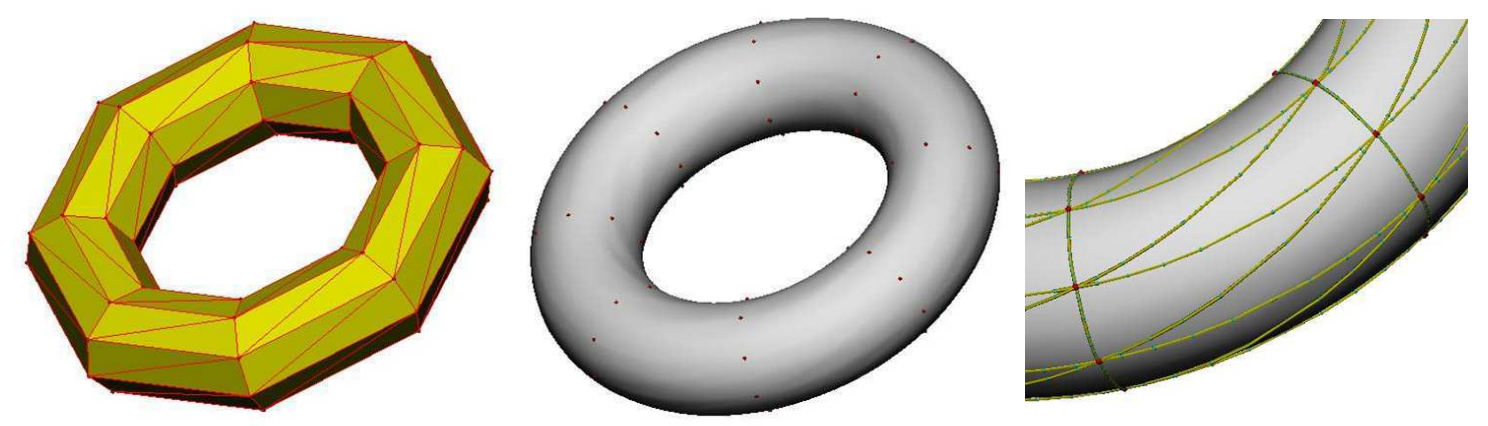

Figure 14: Torus example. from left to right: input mesh, surface with interpolated vertices, zoom with boundary curve's control polygons.

In the rest of this section we show the results of our new interpolation method on several triangular meshes with irregular features, like small and long edges joining at a common vertex, or "dirty" and equilateral triangles joining along a common edge. These features are known for causing unfair surfaces. For each mesh we compare the results of our new method with those obtained by the earlier methods based on a regular n-gone choice of the tangents around the interpolated vertices.

Figure 15 shows left a mesh with regular features: it is an open symmetric mesh with vertices of valence 4,5 , and 6 ; the edges common to the central vertex have all the same length, and all triangles are more or less equiangular. The right part of figure 15 shows the mesh resulting from the translation of the central vertex. Flat triangles appear, and short edges join to long edges at the central vertex. Figure 16 shows the interpolating surface of these two meshes with the previously introduced 4-split method [12]. Each surface is shown twice: with and without the macro-patch boundary curves. While the surface obtained for the regular mesh is acceptable (figure 16-left), the result for the irregular mesh has an unpleasant shape (figure 16-right). Note in particular the overshooting undulations of the surface along the short edges which are joined with the central vertex (figure 16-right). This behavior is due to the fact that the lengths of all tangents at the central vertex are the same. They can't adapt to the length of the curves. Figure 17 shows the results for the new method. For both meshes, the interpolating surface has a nice shape, unwanted 
undulations are avoided.

The next example is a closed mesh with artificially created heavy irregularities. Figure 18 shows two different views we want to focus on. The resulting interpolating surfaces obtained with the early method, fig. 19, are then compared with the results of the new method, fig. 20. Again, unwanted undulations are avoided with the new method and globally fair surfaces are obtained. It is particularly remarkable how the macro-patch boundary curves are able to smoothly follow the edges, because of the free choice of the first derivatives and the ability to vary their lengths around a vertex. On the contrary, the regular n-gone choice of the first derivatives in the old method is clearly the reason why macro-patch boundary curves - and therefore the surface itself - has unwanted undulations (see fig. 19).

In comparison to these result let us also show in fig. 21 the result obtained with one of the two triangular interpolatory subdivision surface schemes: the butterfly subdivision scheme [5]. Again the shape of the surface is clearly not acceptable for this input mesh.
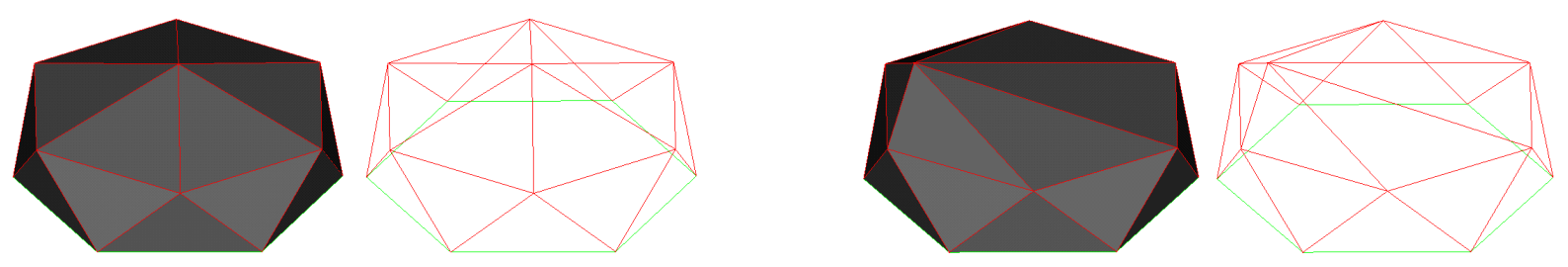

Figure 15: left: regular mesh, right: deformed mesh. both meshes are shown with flat shading and wire frame, they are open meshes.
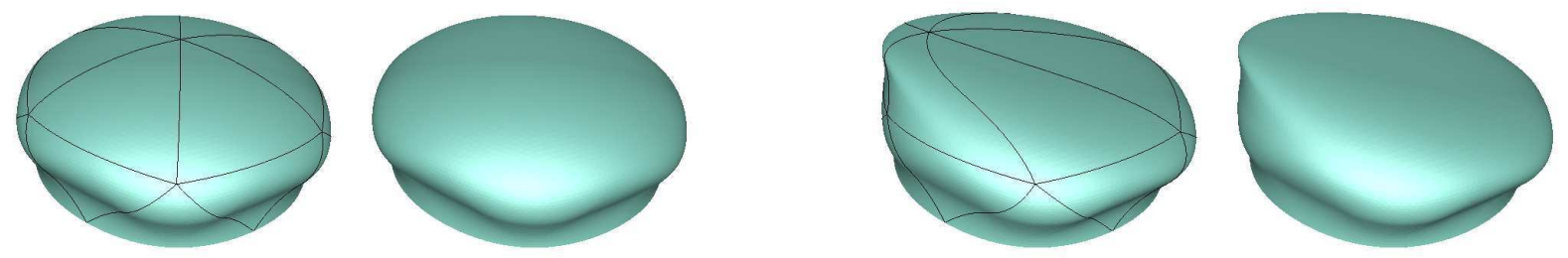

Figure 16: interpolation with affine map of tangents.
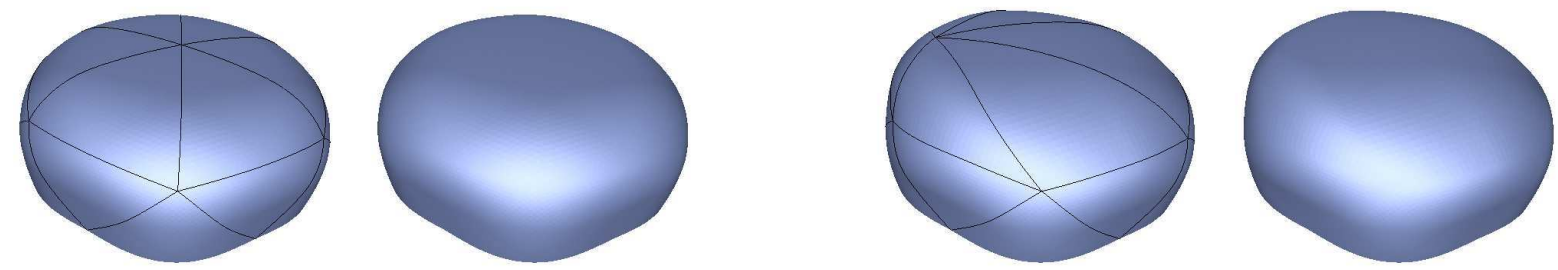

Figure 17: interpolation with arbitrary choice of tangents. 

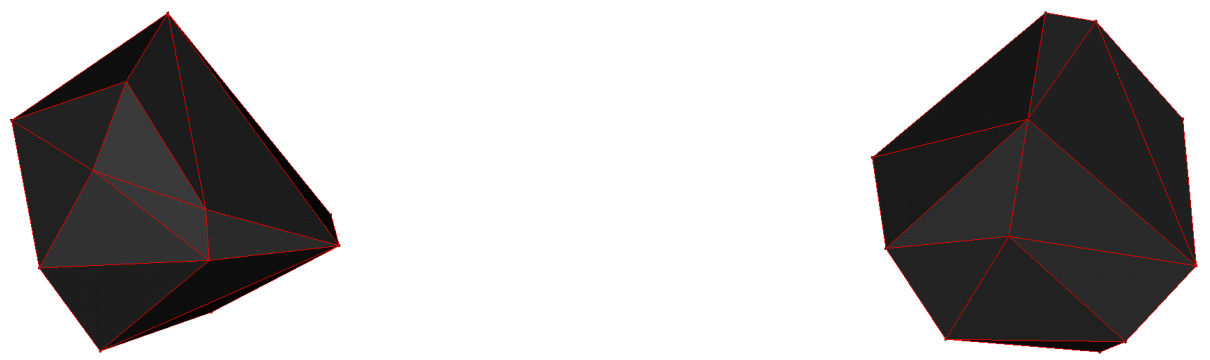

Figure 18: mesh with irregular vertices.
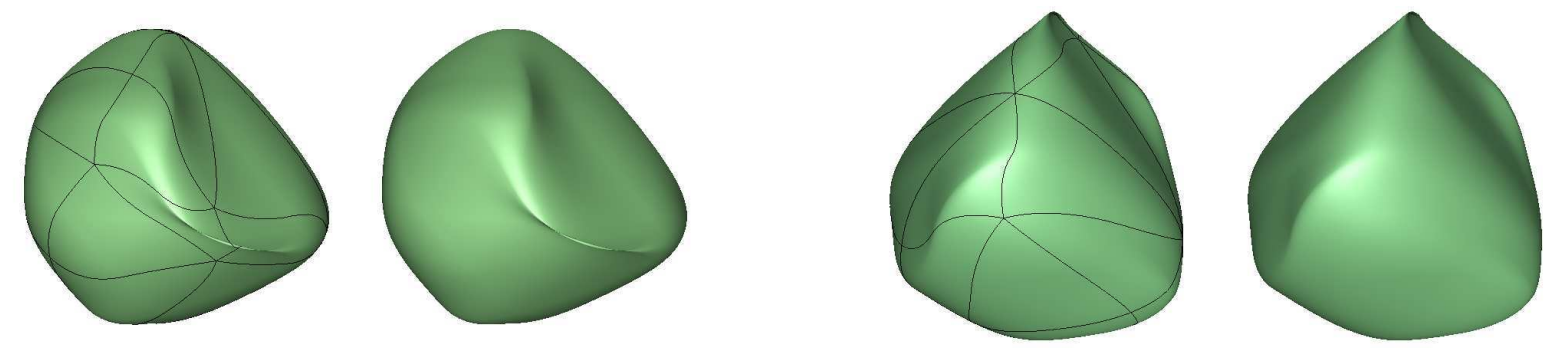

Figure 19: interpolation with affine map of tangents.
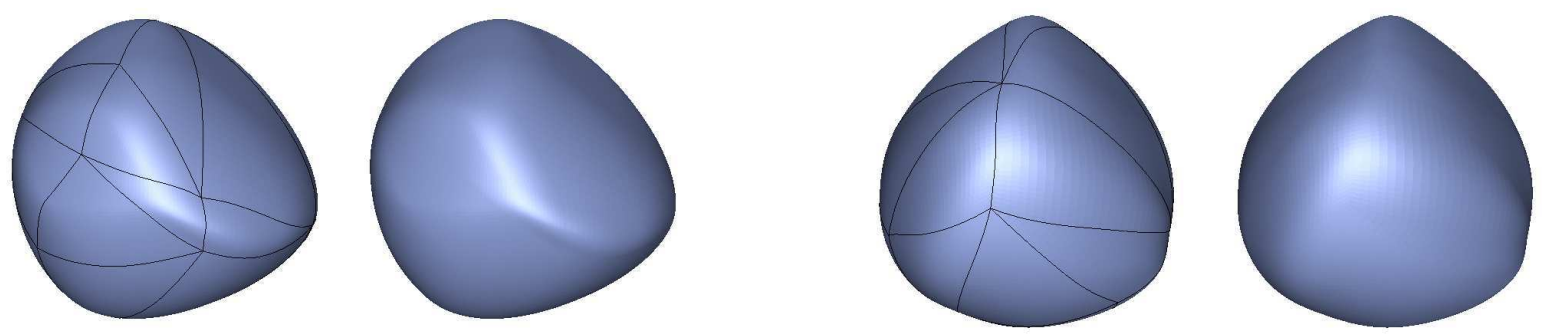

Figure 20: interpolation with arbitrary choice of tangents.

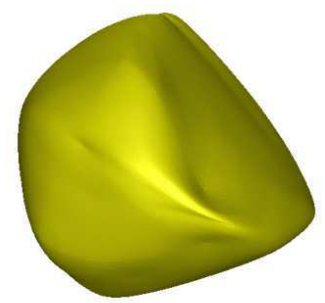

Figure 21: interpolation with the butterfly subdivision scheme. 

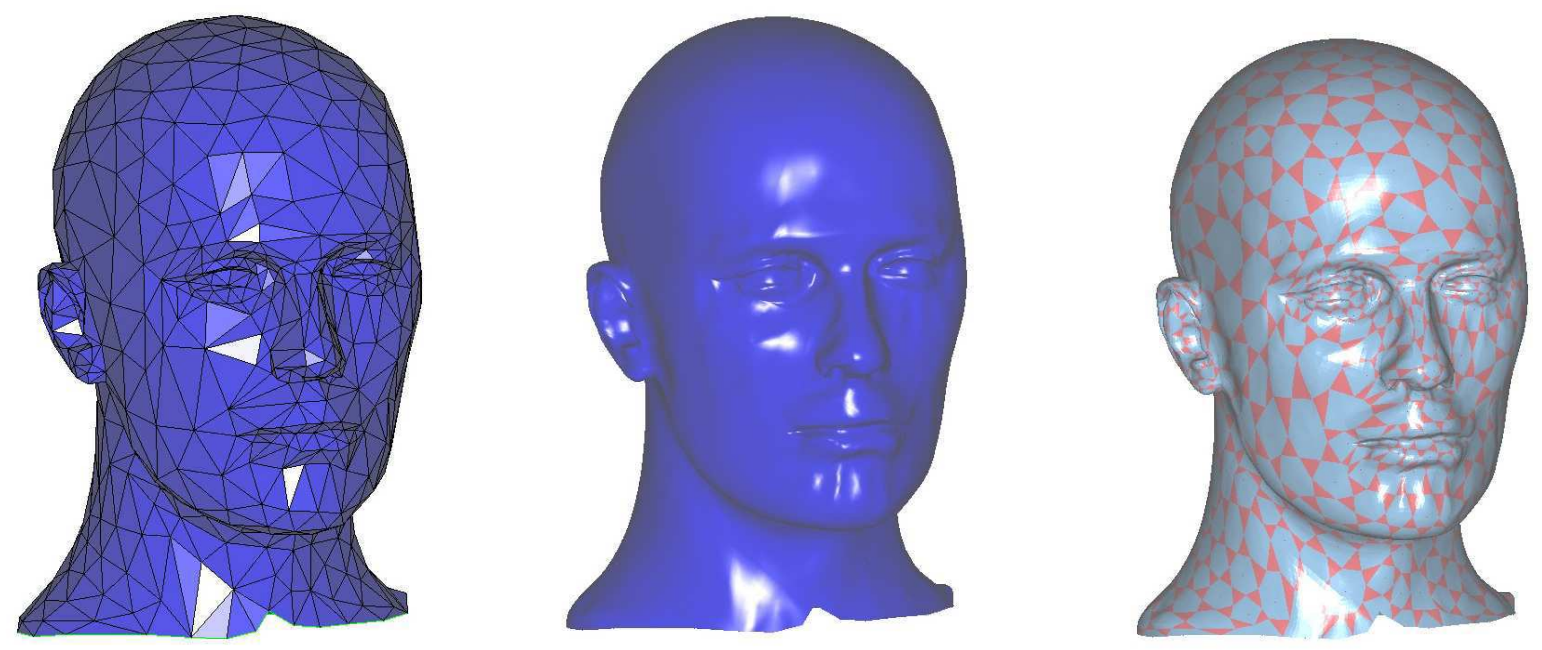

Figure 22: Mannequin, mesh left, $G^{1}$ surface middle, control-polygons right.
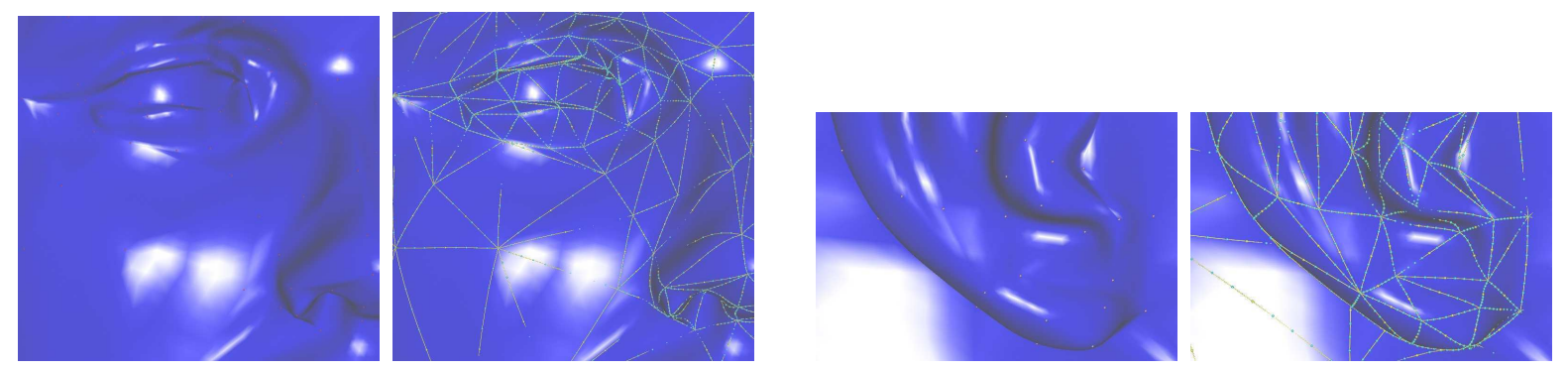

Figure 23: Zoom of the mannequin, without and with control-polygons of boundary curves.

Figure 22 and 23 show the result of our interpolating scheme on a more complex data set, the mannequin data set (courtesy University of Washington). The right part of figure 22 shows the control-nets of the quintic Bézier patches. The central patch of each macro-patch is colored in red. Figure 23 shows two details of the mannequin interpolating surface, without and with the control-polygon of the boundary curves. Note again the overall smoothness and how the boundary curves smoothly follow the edges of the input mesh. This is possible only because of the free choice of the first derivatives.

\section{CONCLUSION}

We have introduced a new method for interpolating 2-manifold triangular meshes with a parametric surface composed of Bézier patches of degree 5 .

While previous similar schemes enforced a regular n-gone choice of the first derivatives at the interpolated vertices, this new method allows a completely free choice of these tangents vectors. We have shown how to derive first and second derivative informations at the interpolated vertices and across the boundary curves between interpolated vertices, such that a polynomial interpolant of low degree can be found. In comparison with previous similar schemes, this new method allows to find pleasing shapes, without unwanted 
undulations, even if the interpolated mesh has non regular features, e.g. short and long edges joining at a common vertex, or flat and big triangles joining along a common edge. Future work will include in particular the refinement of this interpolation scheme. Based on the fact that our method utilized a regular 4-split of the input triangles, we will show that our interpolation scheme is refinable: we will prove that applying our interpolation scheme on a carefully subdivided triangulation yields the same interpolating surface, in other words the interpolation scheme is invariant under subdivision. We will build a multiresolution modeling scheme for the design and edition of complex shapes at different levels of detail.

\section{Acknowledgments}

We would like to thank the TVCG reviewers for their helpfull comments.

This work was partially supported by the European Community 5-th framework program, with the Research Training Network MINGLE (Multiresolution IN Geometric modELing, HPRN-1999-00117).

\section{REFERENCES}

[1.] Barnhill R., Brown J., Klucewicz I., A new twist in CAGD, Computer Graphics and Image Processing, 1978.

[2.] Bajaj C., Smoothing polyhedra using implicit algebraic splines, Computer Graphics 26 (2) (1992), 79-88.

[3.] Brunet P., Increasing the smoothness of bicubic spline surfaces, In R. Barnhill and W. Böhm (eds.) Surfaces in CAGD'84, North-Holland (1985).

[4.] Du H.W., Schmitt F., CAD 22 (9), (1990), 556-573.

[5.] Dyn N., Levin D., Gregory J.A., A butterfly subdivision scheme for surface interpolation with tension control, ACM Transactions on Graphics 9 (1990), 160-169.

[6.] Farin G., A construction for visual $\mathrm{C}^{1}$ continuity of polynomial surface patches, Computer Graphics and Image Processing 20 (1982), 272-282.

[7.] Farin G., Curves and Surfaces for Computer Aided Geometric Design, Academic Press, New York, 4th edition, (1997).

[8.] Gregory J.A., N-sided surface patches, in The Mathematics of Surfaces J. Gregory (ed.), Clarendon Press, Oxford (1986), 217-232.

[9.] Hagen H., Geometric surface patches without twist constraints, Computer Aided Geometric Design 3 (1986), 179-184.

[10.] Hagen H., Schulze G., Automatis smoothing with geometric surface patches, Computer Aided Geometric Design 4 (1987), 231-236. 
[11.] Hagen H., Pottmann H., Curvature continuous triangular interpolants, in Mathematical Methods in Computer Aided Geometric Design Tom Lyche, Larry L. Schumaker (eds.), Academic Press, New York (1989), 373-384.

[12.] Hahmann S., Bonneau G-P., Triangular $\mathrm{G}^{1}$ interpolation by 4-splitting domain triangles, Computer Aided Geometric Design 17 (2000), 731-757.

[13.] Hahmann S., Bonneau G-P., Taleb R., Smooth irregular mesh interpolation, in Curve and Surface Fitting: Saint-Malo 1999 Albert Cohen, Christophe Rabut, and Larry L. Schumaker (eds.), Vanderbilt University Press, Nashville, (2000), 237-246.

[14.] Jensen T., Assembling triangular and rectangular patches and multivariate splines, in Geometric Modeling: Algorithms and new Trends, G. Farin (ed.), SIAM (1987), 203-220.

[15.] Loop C., A G ${ }^{1}$ triangular spline surface of arbitrary topological type, Computer Aided Geometric Design 11 (1994), 303-330.

[16.] Mann S., Surface approximation using geometric Hermite patches, Ph.D. dissertation, University of Washington, (1992).

[17.] Mann S., Loop C., Lounsbery M., Meyers D., Painter J., DeRose T., Sloan K., A survey of parametric scattered data fitting using triangular interpolants, in Curve and Surface Design, H. Hagen (ed.), SIAM (1992), 145-172.

[18.] Neamtu M., Pluger P., Degenerate polynomial patches of degree 4 and 5 used for geometrically smooth interpolation in $\mathbb{R}^{3}$, Computer Aided Geometric Design 11 (1994), 451-474.

[19.] Nielson G., A transfinite, visually continuous, triangular interpolant, in Geometric Modeling: Algorithms and new Trends, G. Farin (ed.), SIAM (1987), 235-246.

[20.] Nowacki H., Reese D., Design and fairing of ship surfaces, In R. Barnhill and W. Böhm (eds.), Computer Aided Geometric Design, North-Holland, (1982), 121-134.

[21.] Peters J., Smooth interpolation of a mesh of curves, Constructive Approximation 7 (1991), 221-246.

[22.] Piper B.R., Visually smooth interpolation with triangular Bézier patches, in Geometric Modeling: Algorithms and new Trends, G. Farin (ed.), SIAM (1987), 221-233.

[23.] Selesnick S., Local invariants and twist vectors in CAGD, Computer Graphics and Image Processing 17, (1981), 145-160.

[24.] Shirman L.A., Séquin C.H., Local surface interpolation with Bézier patches, Computer Aided Geometric Design 4 (1987), 279-295.

[25.] Van Wijk J.J., Bicubic patches for approximating non-rectangular control meshes, Computer Aided Geometric Design 3 (1986), 1-13. 